\title{
Importance of the Hartebeesthoek Radio Astronomy Observatory for the VLBI network
}

\author{
D. Mayer, J. Böhm, L. Combrinck, J. Botai, S. Böhm
}

July 13, 2014

\begin{abstract}
The Hartebeesthoek Radio Astronomy Observatory (HartRAO) is the only Very Long Baseline Interferometry (VLBI) station in Africa, its importance to the current and future network is discussed in the presented analysis. In order to quantify the contribution of the HartRAO station to VLBI products, different approaches (e.g. comparison of number of observations to sources, formal errors of EOP and baseline length repeatabilities) are analysed. Two datasets were used, CONT08 and IVS-R1 sessions from the years 2011 and 2012. The results suggest that the station is of high importance to the current and future global VLBI network. In particular, HartRAO is crucial for maintaining the ICRF on the Southern Hemisphere; it is also one of the most important stations for the estimation of polar motion and nutation (e.g. when HartRAO is excluded from different networks the formal error of nutation increases by about $50 \%$ ). The quality of the station coordinates of remote southern stations such as TIGOCONC is also heavily dependent on HartRAO.
\end{abstract}

Keywords: VLBI, HartRAO, CONT08 


\section{Introduction}

Very Long Baseline Interferometry (VLBI) is a space geodetic technique that observes radio sources at the edge of the observable universe (quasars) in order to determine certain parameters of the system Earth. For example, the VLBI observations contribute to the establishment and maintenance of the International Terrestrial Reference Frame (ITRF) (Altamimi et al., 2011) and it is the only technique that is able to maintain the International Celestial Reference Frame (ICRF) (Fey et al., 2009). Since the VLBI technique is based on observing extragalactic radio sources from the Earth's surface, the VLBI measurements can be used to determine the orientation of the Earth in space. Moreover, VLBI is the only technique that allows simultaneous estimation of all five Earth Orientation Parameters (EOP), namely two angles for polar motion, two angles for nutation and the Earth rotation angle (dUT1). (Schuh and Böhm, 2013)

The VLBI observable is the difference in arrival time of a radio signal at two observing stations. Since the radio sources observed are billions of light years away, the emitted electromagnetic wave is assumed to be planar by the time it arrives at the observing stations. Figure 1 shows the measurement principle, where (1) and (2) are the stations, $\mathbf{s}$ is the direction of the radio source and $\mathbf{b}$ ' the baseline vector. (Schuh and Böhm, 2013)

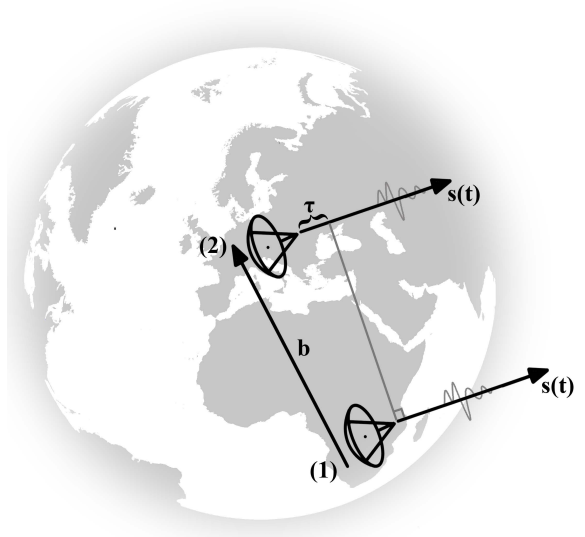

Fig. 1 VLBI measurement principle 
The main observable of the VLBI technique is the time delay $\tau$, it represents the elapsed time between recording time $t_{1}$ and $t_{2}$, and can be calculated using the scalar product divided by the velocity of light $c$, see Equation (1).

$$
\tau=\left(t_{1}-t_{2}\right)=-\frac{\boldsymbol{b}^{\prime} \cdot \boldsymbol{s}}{c},
$$

with $\boldsymbol{b}^{\prime}$ and $s$ in the celestial reference frame. For more details on VLBI, see Sovers et al. (1998) or Schuh and Böhm (2013).

The VLBI technique is rather old (developed in the 1960s), and many stations are using outdated equipment. To tackle this issue the International VLBI Service for Geodesy and Astrometry (IVS) (Schuh and Behrend, 2012) established a working group to determine the future of VLBI. The IVS initiated a plan to upgrade the current network (building new telescopes, establishing new sites etc.) with the aim of achieving $1 \mathrm{~mm}$ accuracy for station position and 0.1 $\mathrm{mm} /$ year for velocities. This new network is called VGOS (previously referred to as VLBI2010), an acronym which reflects VLBI within the framework of the Global Geodetic Observing System (GGOS) (Petrachenko et al., 2009). One of the main issues is the unequal distribution of sites over the globe. For economical and political reasons most stations are situated in the Northern Hemisphere. In recent years new telescopes were developed in Australia and New Zealand (Lovell et al., 2013). However, the African continent still has a very poor VLBI network geometry, with only one station (Hartebeesthoek Radio Astronomy Observatory - HartRAO) located near Johannesburg, South Africa (Latitude: $25^{\circ} 53^{\prime} 27.1^{\prime \prime}$ South, Longitude: $27^{\circ} 41^{\prime} 12.7^{\prime \prime}$ East) (Nicolson, 1995). Since VLBI telescopes are expensive, new sites have to be chosen with care, therefore an evaluation of the importance of existing sites is of high relevance to determine their future use. 


\section{Data and analysis strategy}

The presented analysis considered VLBI observations during the continuous VLBI experiment in 2008, hereafter CONT08 and IVS-R1 sessions where HartRAO participated during the years 2011 and 2012.

The CONT08 project was an uninterrupted VLBI observation session of 15 days. A global network of 11 VLBI stations was selected, with the aim of calculating EOP with the highest precision and accuracy. It took place from the 12th to the 26th of August 2008. In preparation for CONT08 the stations underwent extensive testing to make sure that all the hardware worked correctly (Behrend and Nothnagel, 2013).

The purpose of the IVS-R1 sessions is to estimate EOP solutions on a weekly basis (every Monday and Thursday). Every network consists of 8 stations and, to keep the turnaround time (the time from the measurement to the result) short, it is obligatory to send the recorded data to the correlator as fast as possible (Behrend and Nothnagel, 2013).

The Department of Geodesy and Geoinformation at the Vienna University of Technology has developed new analysis software for VLBI, the Vienna VLBI Software (VieVS) (Böhm et al., 2012). VieVS was used to process the CONT08 as well as the IVS-R1 sessions with the latest models for ocean tidal loading, atmospheric tidal loading etc., according to the Conventions of the International Earth Rotation and Reference Systems Service (IERS) (Petit and Luzum, 2010). In the VLBI analysis using VieVS, station coordinates, EOP, atmospheric and clock parameters were estimated using the default parameterisation of VieVS, i.e. EOP and station coordinates were estimated as constant parameters per 24 hours, and the datum for the station coordinates was realized with a No-NetTranslation and a No-Net-Rotation (NNT/NNR) condition. 


\section{Evaluating the Importance of the HartRAO station}

\subsection{Importance of HartRAO for the ICRF}

The ICRF is one of VLBI's major products. It requires an evenly distributed sky coverage, which can only be achieved with stations located on different latitudes. In these experiments the ICRF2 catalogue was used as basis. The ICRF2 has 697 sources north and 520 sources south of the Equator when only non VLBA Calibrator Survey (VCS) sources are considered (Fey et al., 2009). In the CONT08 experiment 80 sources were used, 52 in the Northern Hemisphere and 28 on the Southern Hemisphere.

To evaluate the importance of HartRAO on the observation of sources (conducted during the CONT08 project) the total number of observations was calculated on a source-by-source basis for the entire CONT08 time span. This was done twice, the first time including all of the observations in the original schedule, and the second time excluding observations with HartRAO. Figure 2 plots this ratio as a function of the declination of the sources. It is obvious that omitting HartRAO dramatically reduces the number of observations of southern sources. Two of the southern sources are not observed at all when HartRAO is absent.

The compared number of observations in Figure 2 (y axis) is a summation of all 15 CONT08 session once with, and once without the station HartRAO.

Figure 3 depicts a similar analysis, here the data from the IVS-R1 sessions were used. In order to get a better representation the sources were divided into $10^{\circ}$ declination intervals, their observations were summed up and plotted in a histogram. This was done for all the sessions once with and once without HartRAO. One can see that when HartRAO is excluded from the IVS-R1 sessions the number of observations in the Southern Hemisphere, which is already low, decreases by about $35 \%$. 


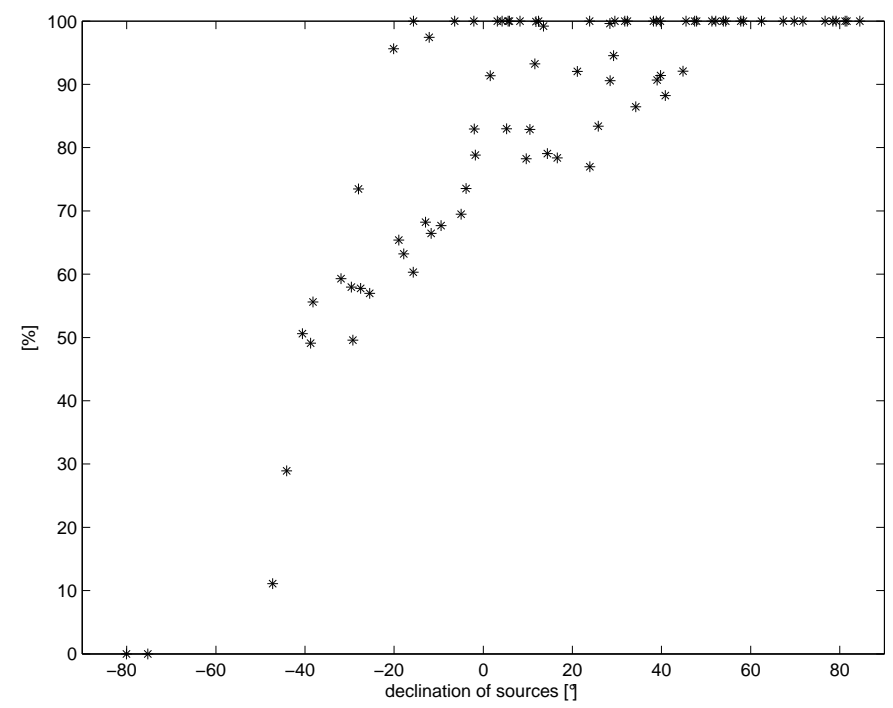

Fig. 2 Sources of CONT08 network - comparison of number of observations with and without HartRAO [\%] and declination of the sources

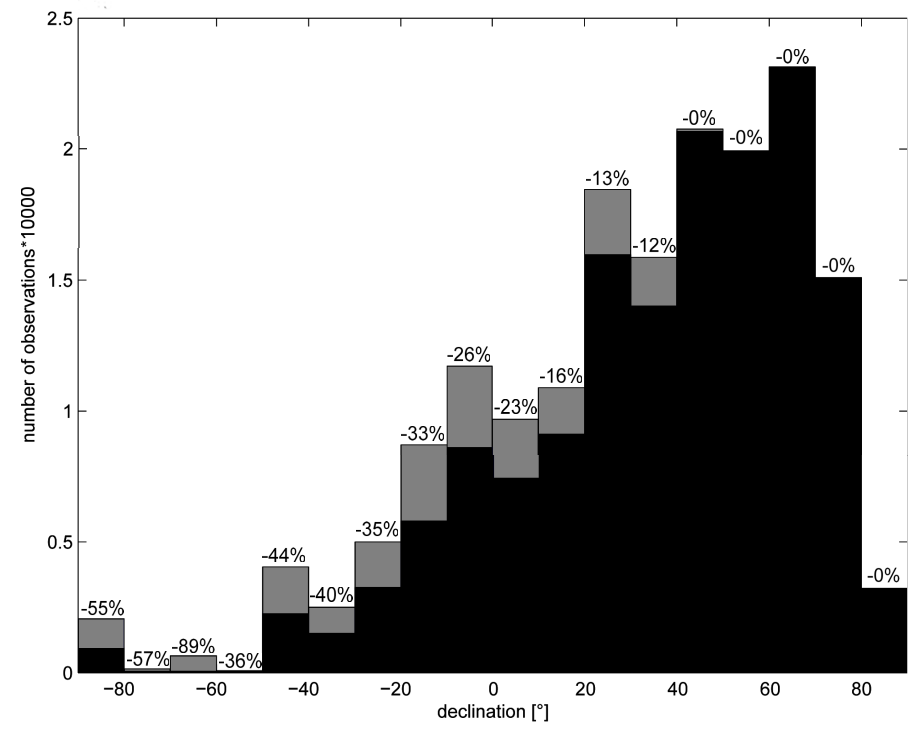

Fig. 3 Histogram of source observations with and without HartRAO from the IVS-R1 sessions divided in $10^{\circ}$ declination intervals. The difference between the network with and without HartRAO is indicated in grey. We also provided a percentage value to indicate the loss of observations when HartRAO is excluded from the network 
The results from the analysis of both data sets suggest that HartRAO is a crucial station for maintaining the ICRF. In particular, sources south of the Equator are highly dependent on observations done at HartRAO in existing schedules. Since real data are used in this analysis the schedule is not optimal, hence the results are too pessimistic.

\subsection{Formal error and repeatability of EOP}

Hase (2010) demonstrated that the formal error of the EOP is a good indicator that could be used for quantifying the importance of a specific VLBI site. In this section the formal error and the repeatability of the EOP calculated once with and once without a particular VLBI site are discussed.

All 15 sessions from the CONT08 experiment, each session representing one day, were processed with the default parameterisation mentioned in Chapter 2 . To estimate the importance of each station, the network was analysed 11 times and each time a different station was removed (all observations with this station were removed on a station-by-station basis). The resulting EOP were then statistically examined and compared. Parameters in this section might be too pessimistic, because removing a station results in an suboptimal schedule. For an accurate result, the same experiment has to be conducted several times with a different schedule (each time one station is removed before making the schedule). Unfortunately, such data were not available, therefore this is a simulated case where the station was removed from the schedule. However, a similar analysis with optimised schedules and simulated observations can be found in Chapter 3.4 .

Table 1 lists the average (over all 15 sessions) formal errors of the EOP (the $\mathrm{x}$ and $\mathrm{y}$ coordinate of polar motion is denoted as xpol and ypol respectively, the rotation angle is denoted as dut 1 and the $\mathrm{x}$ and $\mathrm{y}$ coordinates of nutation are denoted as nutdx and nutdy respectively). These values (average formal errors) represent the geometry of the network and are therefore a good measure for the comparison of VLBI networks. In theory the geometry is best, when as 
many stations as possible are equally distributed over the Earth's surface. This approach can be confirmed by looking at Table 1, where it can be seen that the average formal error is the smallest when all stations are used. The data suggest that, in general, remote telescopes (in this case outside of Europe) have a high impact on the precision of the EOP.

Nothnagel et al. (1988) proved that the north-south component of baselines is the most sensitive to polar motion. It is therefore crucial to include stations in high and low latitudes, when pole coordinates are estimated. The data derived from the CONT08 sessions confirm these findings.

To get long east-west baselines, and hence a good estimation of dUT1, stations at different longitudes are essential. The results in Table 1 clearly indicate that remote stations, such as TSUKUB32 and KOKEE, have a higher impact on dUT1 than other comparable telescopes in regions with a high density of stations. Without KOKEE the formal error of dUT1 doubles. The results from Table 1 suggest that when HartRAO is excluded from the network the formal error in the y coordinate of the polar motion doubles. Table 1 also lists the nutation and the results imply that HartRAO is by far the most important station of the CONT08 network for the estimation of nutation in the existing schedules.

Table 2 contains the standard deviation (repeatability) of the estimated parameters w.r.t. the a-priori values (IERS $08 \mathrm{C} 04$ ). In an ideal world the standard deviation of the estimated EOP would be the best (smallest) if all stations are observing. Here, however, some parameters show smaller standard deviations when certain stations are removed. This is due to the fact that data from some stations might be less accurate or corrupted. Nevertheless the importance of HartRAO for the estimation of polar motion and nutation can be seen clearly. When HartRAO is removed from the network, the standard deviation of the $\mathrm{y}$-coordinate of the polar motion as well as the $\mathrm{x}$-coordinate of the nutation almost triples. It has to be noted that the standard deviation was derived with only 15 sessions and is therefore prone to outliers.

A similar analysis was also conducted with the data from the IVS-R1 ses- 


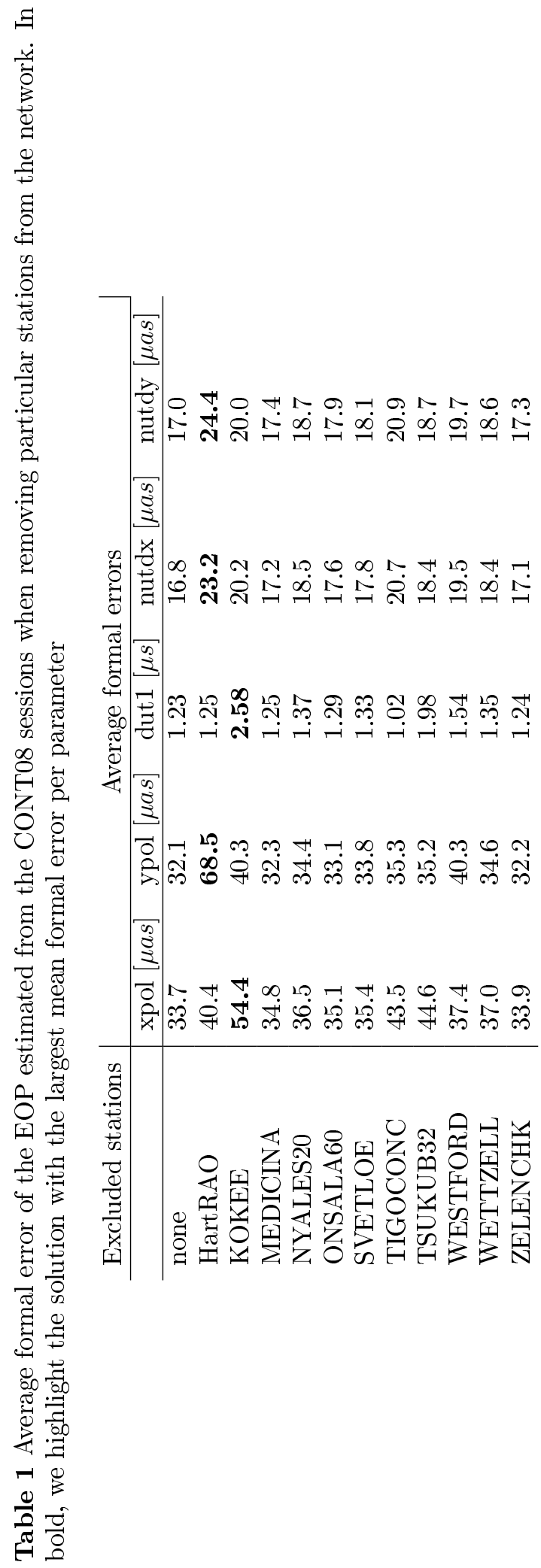




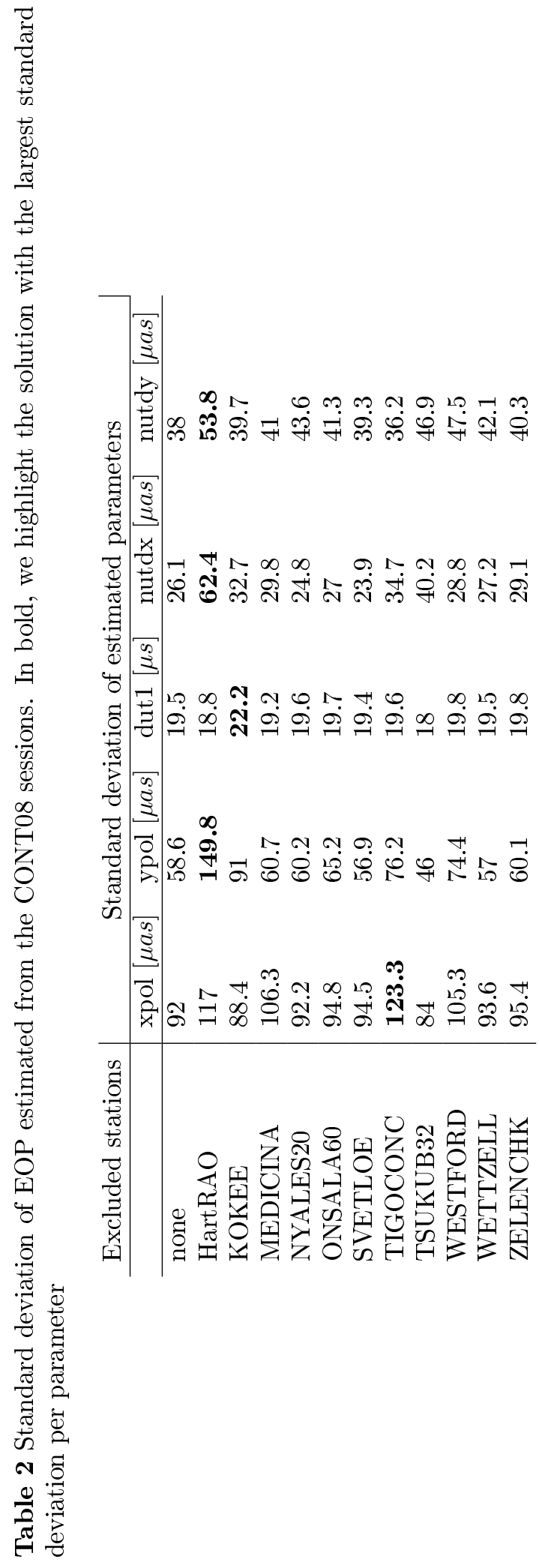


sions from the beginning of 2011 until the end of 2012. Since the networks used here are not consistent (their geometry changes) the examination was only done for the station HartRAO. The same parameterisation was used, therefore results can be compared. The data were once processed with and once without HartRAO. As mentioned previously the results are too pessimistic due to imperfect schedules.

In Figure 4 the formal errors of the EOP are depicted. The results suggest that the EOP precision gets worse when HartRAO is excluded, in particular the polar motion and nutation are affected. All following percentage information in this paragraph was obtained by calculating the precision change (how much larger does the formal error get if HartRAO is excluded) for each session and in a second step the average error increase was determined. The median is written in brackets next to the average value. Excluding HartRAO from the network leads to an increase of the formal error of polar motion by $55 \%$ (52\%) for the $\mathrm{x}$ part and by $142 \%$ (124\%) for the y part. The nutation is affected to a similar extent, the formal error increases by $55 \%$ (50\%) for the $\mathrm{X}$ part and $60 \%(55 \%)$ for the Y part. One can also see from this data that excluding HartRAO yields only small changes, a $23 \%$ (0\%) decrease was observed for the formal error of dUT1. All findings agree with the results for the CONT08 campaign.

Figure 5 depicts the estimates of the EOP w.r.t. the a-priori values (IERS 08 C04). Black lines represent EOP estimates calculated with unmodified IVSR1 sessions (with HartRAO). The grey line correspondes to the same sessions but HartRAO was excluded from the schedule beforehand. To compare these two results the standard deviation of the EOP estimates (repeatability) was calculated .The smallest difference occurs between the dUT1 standard deviations (an increase of 33\%). Similar to the previous findings the polar motion (103\% increase for the $\mathrm{x}$ part and $120 \%$ for the $\mathrm{y}$ part) and nutation (37\% increase for the $\mathrm{x}$ part and $122 \%$ for the $\mathrm{y}$ part) is affected greatly when HartRAO is removed from the network. 

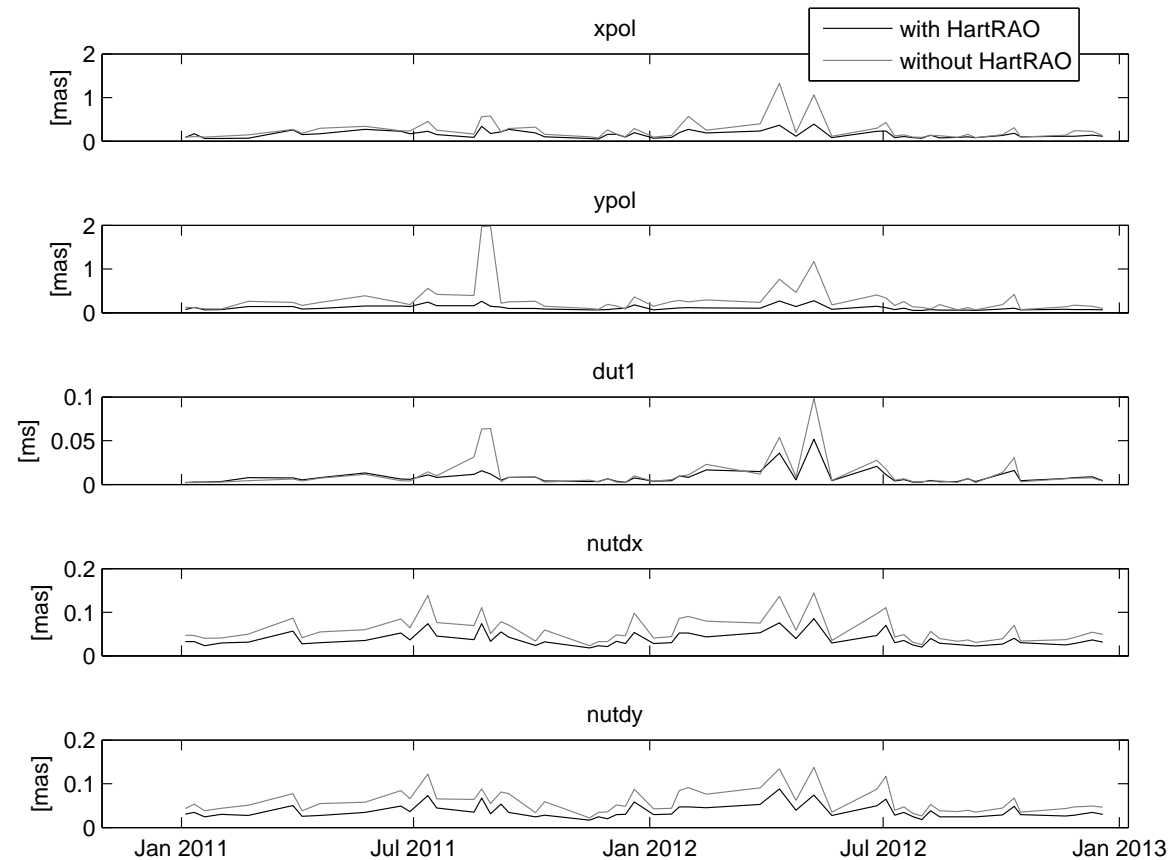

Fig. 4 Formal error of the EOP estimated from IVS-R1 sessions once analysed with and once without the station HartRAO
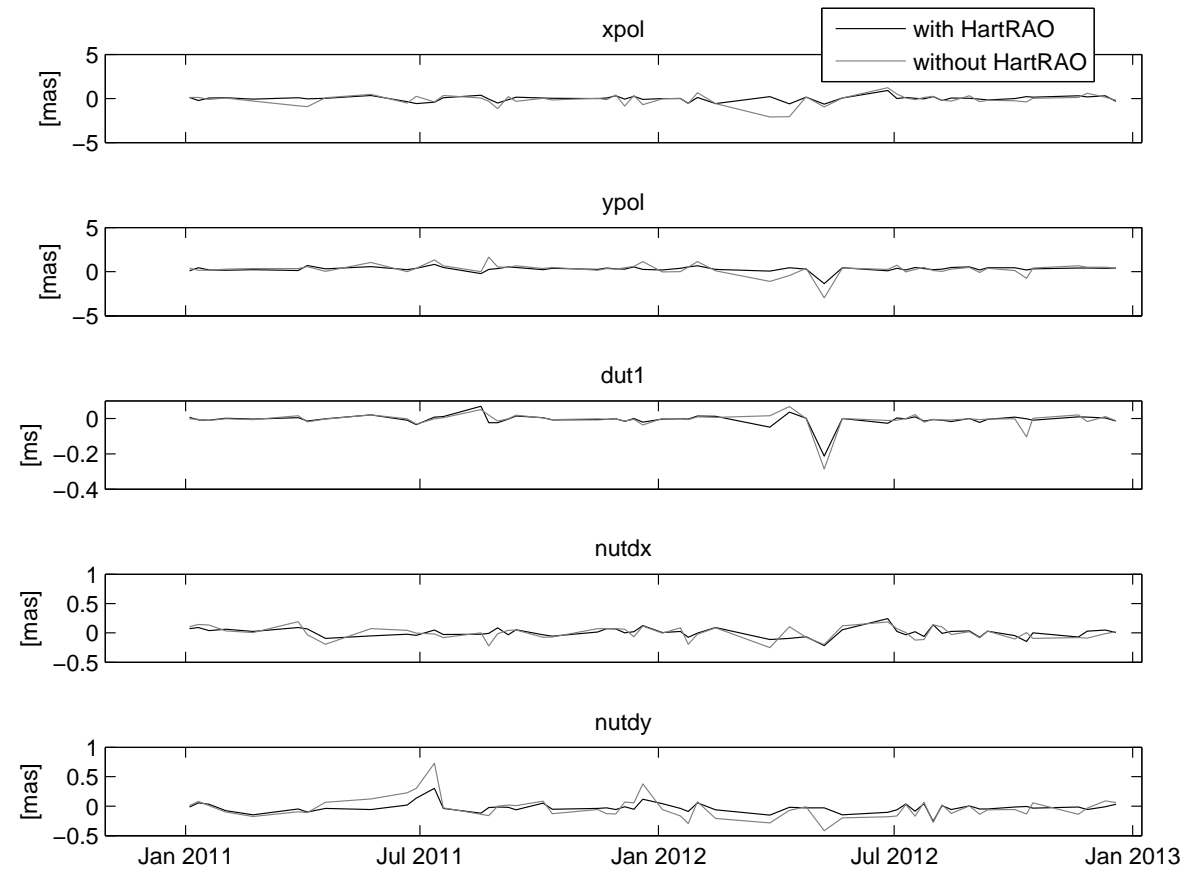

Fig. 5 EOP estimates w.r.t. IERS 08 C04 series from the IVS-R1 sessions once analysed with and once without the station HartRAO 


\subsection{Baseline length repeatability}

A very important part of VLBI is the estimation of station coordinates. Comparing baseline length scatter is particularly important since the baseline is independent from the Earth's orientation. In order to quantify the importance of HartRAO on the estimated VLBI station coordinates, the baseline length repeatability plot is examined. This plot illustrates the weighted standard deviation (repeatability) of every baseline of the network. To get the weighted standard deviation, baselines from all sessions of the CONT08 experiment as well as baselines from the IVS-R1 sessions were taken into account. The inverse of the formal error of each baseline was used as a weight. Since baselines with less observations have a higher formal error the weighting accounts for accuracy loss due to less observations. The mean (average baseline length) and the weighted standard deviation after removing a linear trend were calculated.

The repeatability of the baselines of the CONT08 experiment, once calculated with and once without HartRAO, is depicted in Figure 6. It can be seen that an exclusion of HartRAO has almost no effect on shorter baselines (mainly Europe), but the repeatability of longer baselines gets worse (up to approximately $5 \mathrm{~mm}$ ) when HartRAO is removed. In particular the repeatability of baselines with TIGOCONC (dots in the upper right corner) are getting larger when HartRAO is removed from the network.

The repeatability of baselines estimated once with and once without HartRAO from the IVS-R1 data is depicted in Figure 7. The results are similar to those for CONT08. HartRAO has almost no effect on shorter baselines but on longer baselines. Hence, more remote stations are affected greatly by the removal of HartRAO in existing schedules. In particular TIGOCONC benefits from HartRAO, which is due to the high number of observations between HartRAO and TIGOCONC in the existing schedules. In order to get reasonable results the station drift was removed beforehand, the earthquake in Japan (March 11, 2011) was corrected and only baselines which were estimated at least 5 times (higher thresholds were also used which resulted in similar outcomes) 


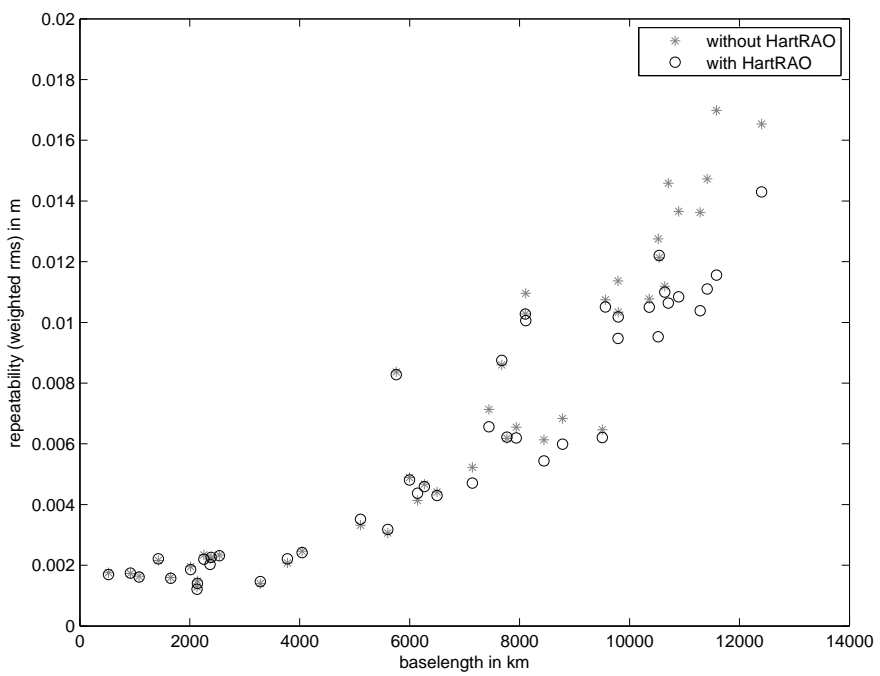

Fig. 6 Baseline length repeatability (weighted rms) plot of the CONT08 network, with and without HartRAO

were taken into account.

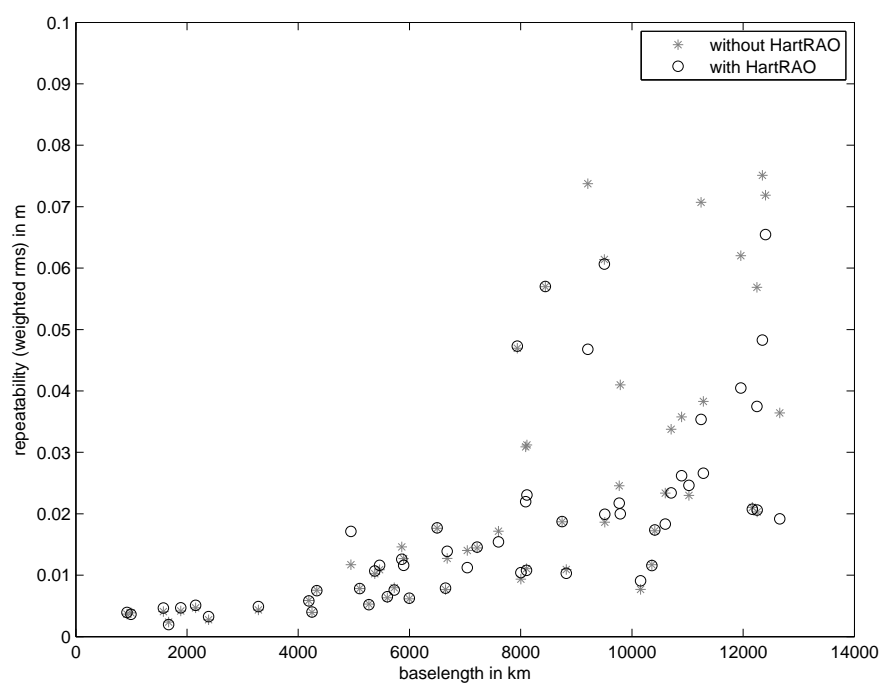

Fig. 7 Baseline length repeatability (weighted rms) plot of the IVS-R1 sessions of the year 2011 and 2012 with and without HartRAO 


\subsection{VGOS simulated network}

In this chapter the importance of the station HartRAO for the new VGOS network is discussed. The effects of three different telescopes placed at the site are analysed. The following telescopes are used for the evaluation:

- Hart15 is already in use at HartRAO, it has a $15 \mathrm{~m}$ diameter and a slewing speed of $2 \mathrm{deg} / \mathrm{s}$ for azimuth and $1 \mathrm{deg} / \mathrm{s}$ for elevation.

- Hart2010 is a VGOS antenna with a diameter of $12 \mathrm{~m}$ and a slewing speed of $12 \mathrm{deg} / \mathrm{s}$ for azimuth and $6 \mathrm{deg} / \mathrm{s}$ for elevation.

- HartTWIN is a twin telescope made out of two VGOS antennas. It is the strongest telescope tested in this examination and should yield the best result.

To evaluate the importance of HartRAO for VGOS a network with 16 stations as suggested by Sun et al. (2014) is used. A network of at least 16 stations was also proposed by the IVS committee (Petrachenko et al., 2009).

Fast slewing VGOS antennas with identical characteristics (no twin telescopes were used) were placed at each site of the network. By using the same telescope at every station in the network the effects of station specific parameters from other stations than Hartebeesthoek, such as slew speed and diameter of the dish, are neutralised.

To see the influence of the three different telescopes on the VLBI results the network is simulated four times: with Hart15, Hart2010 and HartTWIN and once where no telescope was placed at Hartebeesthoek. The date for the $24 \mathrm{~h}$ session was randomly set to 21 of June 2012. Every network in this chapter was analysed using the same parameterisation.

Scheduling parameters:

- Minimal source flux density of $0.3 J y$.

- Sun distance must be more than $15^{\circ}$. 
- Cut off elevation angle of $5^{\circ}$.

- Observations are carried out in $\mathrm{S} / \mathrm{X}$ band, using 14 channels with a bandwidth of $128 \mathrm{MHz}$, a sample rate of $256 \mathrm{MHz}$ and a quantisation of 2 bits. The minimum Signal-to-Noise Ratio (SNR) was set to 15 for S band and 20 for $\mathrm{X}$ band.

- We used the source based scheduling strategy (Sun et al., 2014).

Simulation parameters (Pany et al., 2011):

- Troposphere parameters:

- refractive index structure constant $C_{n}$ of $2.5 \cdot 10^{-7} m^{-\frac{1}{3}}$

- effective height of wet troposphere of $2000 \mathrm{~m}$

- the wind velocity vector towards east of $8 \frac{m}{s}$

- The stochastic variation of the station clock is simulated as a sum of random walk and an integrated random walk. A power spectral density (Allan Standard Deviation; ASD) of $10^{-14} @ 50$ min was used.

- A white noise of $16 p s$ was added to each computed baseline observation.

In order to get a better overview the average formal errors are represented in percentage w.r.t. the average formal error of the network with HartTWIN (this network geometry has the smallest average formal error of the EOP it is considered the ideal case in this scenario) and visualised in Figure 8, e.g. the average formal error of the $\mathrm{x}$ coordinate of polar motion increases by about $40 \%$ - w.r.t. the best solution (VGOS network with HartTWIN) - if no telescope is placed at Hartebeesthoek. The results suggest that upgrading HartRAO to the best telescope available is of high importance for the VGOS network. In particular polar motion and nutation are affected greatly when HartRAO is not equipped with an adequate telescope or even removed from the network. The repeatability of the EOP estimates was calculated as well, but the tendency is not as clear as for the average formal error. 


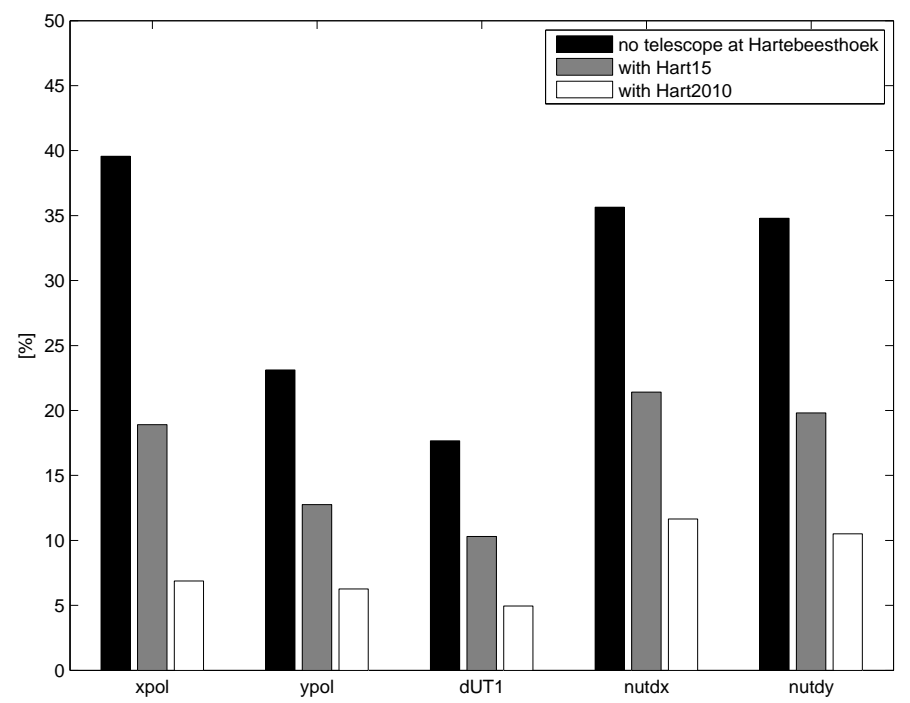

Fig. 8 Average formal error (in percentage) of the EOP from the VGOS network with Hart15, Hart2010 and with no telescope at Hartebeesthoek. The ideal case, e.g. the network with HartTWIN is chosen as a reference (zero line). 


\section{Conclusion}

Different analysis strategies (such as number of radio sources observed by the VLBI network, formal error, baseline length repeatability) have been used to quantify the significance of the HartRAO VLBI station to the global VLBI network. The results suggest that HartRAO is one of the most important stations in the current VLBI network; therefore investing in the future of HartRAO will be of great benefit for the new VGOS network. HartRAO is one of the few VLBI stations capable of observing radio sources in the Southern Hemisphere. Since the number of stations south of the equator is very limited, HartRAO plays a crucial role in maintaining the ICRF. By removing HartRAO from the network the number of source observations decreases with higher southern latitudes.

To evaluate the impact on the EOP estimation, the formal error of the real observations (CONT08 and IVS-R1 observations) was examined. The average formal error of the EOP of the CONT08 experiment was used to evaluate the contribution of each station to the EOP precision using real data. The analysis reveals that HartRAO is especially important for polar motion and nutation. A similar study was done using the IVS-R1 sessions of the years 2011 and 2012. Only the contribution of HartRAO was examined in this data set. This is due to the fact that the network geometry of these sessions is not fixed and therefore a comparable examination can only be done with one station. The results of this data set also suggest that HartRAO is of high importance for estimating polar motion and nutation with the current network.

To quantify the impact of HartRAO on the station coordinate estimation of the network, the baseline length repeatability was examined for the CONT08 experiment and the IVS-R1 sessions. Similar outcomes are obtained with both data sets. HartRAO has almost no influence on the short baselines of the network, but when HartRAO is removed from existing schedules the error increases with longer baseline length, in particular for baselines with TIGOCONC, a station heavily depending on observation with HartRAO. 
Since real data were used for this analysis rescheduling a network without HartRAO was not an option. Therefore the same schedule was used and observations involving HartRAO were removed. This, however, results in a less than optimal schedule for the remaining stations and hence the results in this note might be too pessimistic. To address this issue a second examination was done where schedules were created and observations simulated. The future network with 16 VGOS stations was used for the analysis. The contribution of different telescopes (Hart15, Hart2010 and HartTWIN) at Hartebeesthoek to this network was examined. Based on the outcome of this analysis we recommend upgrading the site with the best telescope feasible. In particular the polar motion and nutation precision is dependent on HartRAO which is due to the fact that baselines with HartRAO have long north-south components and are therefore sensitive to these EOP. A failure of HartRAO has severe effects on the formal errors of EOP. We therefore also recommend to increase the density of VLBI sites on the Southern Hemisphere, in particular in Africa.

The same approach can be used to examine the importance of HartRAO (or any other station or future station) for different networks. That, however, is a subject for further research. 


\section{Acknowledgements}

The authors would like to thank the Hartebeesthoek Radio Astronomy Observatory and the Vienna University of Technology for providing financial support for this work, as well as the University of Pretoria for providing facilities and assistance. David Mayer and Johannes Böhm are grateful to the Austrian Science Fund (FWF) for supporting project P23143-N21 ('Integrated VLBI"). 


\section{References}

Altamimi, Z., Collilieux, X., and Métivier, L. (2011). ITRF2008: an improved solution of the international terrestrial reference frame. Journal of Geodesy, $85(8): 457-473$.

Behrend, D. and Nothnagel, A. (2013). International VLBI Service for Geodesy and Astrometry (IVS). In Report of the International Association of Geodesy 2011-2013 - Travaux de l'Association Internationale de Géodésie 2011-2013, volume 38, pages $364-371$.

Böhm, J., Böhm, S., Nilsson, T., Pany, A., Plank, L., Spicakova, H., Teke, K., and Schuh, H. (2012). The new Vienna VLBI Software VieVS. In Proceedings of the 2009 IAG Symposium, Buenos Aires, Argentina, volume 136, pages 1007-1011. International Association of Geodesy Symposia. 31 August - 4 September 2009 .

Fey, A., Gordon, D., and Jacobs, C. (2009). The Second Realization of the International Celestial Reference Frame by Very Long Baseline Interferometry. IERS technical note 35. Frankfurt am Main: Verlag des Bundesamts für Kartographie und Geodäsie.

Hase, H. (2010). Zur Bedeutung von TIGO zur Bestimmung des ITRF, der EOP, EUREF und GREF. Technical report. Available at http: //www.tigo . cl/documents/100127BedeutungTIGO.pdf.

Lovell, J., McCallum, J., Reid, P., McCulloch, P., Baynes, B., Dickey, J., Shabala, S., Watson, C., Titov, O., Ruddick, R., Twilley, R., Reynolds, C., Tingay, S., Shield, P., Adada, R., Ellingsen, S., Morgan, J., and Bignall, H. (2013). The auscope geodetic VLBI array. Journal of Geodesy, 87(6):527538.

Nicolson, G. (1995). VLBI at the hartebeesthoek radio astronomy observatory. Astrophysics and Space Science, 230:329-339. 
Nothnagel, A., Nicolson, G. D., Schuh, H., Campbell, J., Cloppenburg, H., and Kilger, R. (1988). Radiointerferometric Polar Motion Determination Using a Very Long North-South Baseline. In Babcock, A. K. and Wilkins, G. A., editors, The Earth's Rotation and Reference Frames for Geodesy and Geodynamics, volume 128 of IAU Symposium, page 193.

Pany, A., Böhm, J., MacMillan, D., Schuh, H., Nilsson, T., and Wresnik, J. (2011). Monte carlo simulations of the impact of troposphere, clock and measurement errors on the repeatability of VLBI positions. Journal of Geodesy, $85(1): 39-50$.

Petit, G. and Luzum, B., editors (2010). IERS Conventions 2010. Frankfurt am Main: Verlag des Bundesamts für Kartographie und Geodäsie. IERS Technical Note No. 36.

Petrachenko, B., Niell, A., Behrend, D., Corey, B., Böhm, J., Charlot, P., Collioud, A., Gipson, J., Haas, R., Hobiger, T., Koyama, Y., MacMillan, D., Malkin, Z., Nilsson, T., Pany, A., Tuccari, G., Whitney, A., and Wresnik, J. (2009). Design Aspects of the VLBI2010 System. Progress Report of the IVS VLBI2010 Committee. Technical report. Available at http://adsabs . harvard.edu/abs/2009vlbi.rept....1P.

Schuh, H. and Behrend, D. (2012). VLBI: A fascinating technique for geodesy and astrometry. Journal of Geodynamics, 61(0):68 - 80 .

Schuh, H. and Böhm, J. (2013). Very long baseline interferometry for geodesy and astrometry. In Xu, G., editor, Sciences of Geodesy - II, pages 339-376. Springer Berlin Heidelberg.

Sovers, O. J., Fanselow, J. L., and Jacobs, C. S. (1998). Astrometry and geodesy with radio interferometry: experiments, models, results. Reviews of Modern Physics, 70(4):1393-1454.

Sun, J., Böhm, J., Nilsson, T., Krásná, H., Böhm, S., and Schuh, H. (2014). New 
VLBI2010 scheduling strategies and implications on the terrestrial reference frames. Journal of Geodesy, 88(5):449-461. 